\title{
Self injurious behaviour in Tourette syndrome: correlates with impulsivity and impulse control
}

\author{
C A Mathews, J Waller, D V Glidden, T L Lowe, L D Herrera, C L Budman, G Erenberg, A Naarden, \\ R D Bruun, N B Freimer, V I Reus
}

J Neurol Neurosurg Psychiatry 2004;75:1149-1155. doi: 10.1136/jnnp.2003.020693

See end of article for authors' affiliations .....................

Correspondence to: Dr C A Mathews Department of Psychiatry, University of California, San Diego, 9500 Gilman Dr, 0810, La Jolla, CA 92093-0810.

camathews@ucsd.edu

Received 16 June 2003 Revised

19 September 2003

Accepted

4 November 2003
Background: Self injurious behaviour (SIB), the deliberate, repetitive infliction of self harm, is present in a wide variety of neuropsychiatric disorders, including Tourette syndrome (TS). Although SIB occurs in up to $60 \%$ of individuals with TS, and can cause significant clinical impairment and distress, little is known about its aetiology.

Objective: This study examined the relationship between SIB and other behavioural features that commonly co-occur with TS in nearly 300 subjects with TS participating in three genetic studies. SIB, obsessions, compulsions, tic severity, attention deficit hyperactivity disorder related impulsivity, risk taking behaviours, and rages were systematically assessed in all subjects.

Methods: Using logistic regression, a best fit model was determined for both mild to moderate SIB and severe SIB.

Results: Mild/moderate SIB in TS was correlated with the presence of obsessive and compulsive symptoms such as the presence of aggressive obsessions or violent or aggressive compulsions, and with the presence of obsessive-compulsive disorder and overall number of obsessions. Severe SIB in TS was correlated with variables related to affect or impulse dysregulation; in particular, with the presence of episodic rages and risk taking behaviours. Both mild/moderate and severe SIB were also correlated with tic severity.

Conclusions: This study suggests that mild/moderate and severe SIB in TS may represent different phenomena, which has implications for clinical management of these symptoms.
S elf injurious behaviour (SIB), the deliberate, nonaccidental, repetitive infliction of self harm without suicidal intent, is present in approximately $4 \%$ of the general psychiatric population. ${ }^{1}$ Although SIB can occur in a wide variety of neuropsychiatric disorders, it has been reported most frequently in borderline personality disorder, eating disorders, and psychoactive substance abuse, where the SIB appears to serve the purpose of reducing tension and regulating affect, and in pervasive developmental disorders and organic brain disorders such as Lesch-Nyhan syndrome, where it may be related to neurochemical dysregulation. ${ }^{1-3}$

SIB also occurs with some frequency in movement disorders, most notably Tourette syndrome (TS). ${ }^{3}{ }^{4}$ TS is a neuropsychiatric disorder with onset in childhood that consists of multiple motor and vocal tics and affects approximately 1:2000 people worldwide. ${ }^{56}$ In addition to motor system abnormalities, it is associated with a number of psychiatric comorbidities, most commonly obsessive-compulsive disorder (OCD) and attention deficit hyperactivity disorder (ADHD). Severity of symptoms in TS can range from mild and non-impairing to severe and incapacitating. Although the tics themselves clearly contribute to the severity of the overall disorder, the number and type of comorbid symptoms, including obsessions and compulsions, attentional problems, impulse control problems, and self injurious behaviours are also crucial, and may be more clinically relevant than the tics when assessing impairment. ${ }^{6}$

SIB occurs in up to $60 \%$ of patients with TS, although estimates vary depending on the definition of self injury. ${ }^{3-10}$ A variety of self injurious behaviours have been reported in individuals with TS, including compulsive skin picking, self hitting, lip and other self biting, filing of the teeth, head banging, and eye damage from self poking (reviewed in Robertson $\mathrm{et}^{\mathrm{al}} \mathrm{l}^{3}$ ). Retrospective cohort studies have suggested that SIB is correlated with severity of tic symptoms and with high levels of obsessionality and hostility as measured by a number of subjective rating scales. ${ }^{311}$ In the largest study to date, a retrospective assessment of 3500 individuals with TS collected from all over the world, SIB was present in 14\% of cases (range 4-43\%). Individuals who had TS plus at least one other psychiatric comorbidity had a fourfold increase in SIB, and there was a positive linear relationship between the number of psychiatric comorbidities and the presence of SIB. In this sample, the presence of OCD but not ADHD or mood disorders was highly correlated with SIB. ${ }^{6}$ This study, although compelling because of the large sample size, had two major design limitations: (a) there was no standardised assessment procedure; rather, data were collected retrospectively from medical records in a wide variety of TS clinics using a short data entry form, and $(b)$ the behavioural and diagnostic categories, including SIB and obsessive-compulsive (OC) behaviours, did not have established operational definitions.

In this study, we examined the relationship between TS and SIB in a sample of nearly 300 individuals who were originally investigated in the US and in Costa Rica for genetic studies of TS. As has been suggested by previous studies, we hypothesised that SIB in TS, rather than being a simple manifestation of illness severity, is related to the presence of a combination of comorbid conditions.

SIB in other neuropsychiatric disorders appears to be related to problems with impulse control. ${ }^{12}{ }^{13}$ Impulse dysregulation is seen in a number of neuropsychiatric disorders that affect children with TS, including ADHD,

Abbreviations: ADHD, attention deficit hyperactivity disorder; ASP, affected sibling pair; PGM, population genetic mapping; SIB, self injurious behaviour; TS, Tourette syndrome; TSA, Tourette Syndrome Association; YGTSS, Yale Global Tic Severity Scale; YSRF, Yale Self Report Form 
depression, and bipolar disorder. ${ }^{512} 1415$ However, despite evidence for impulse dysregulation in ADHD and mood disorders, previous studies in TS have indicated that SIB is not correlated with the presence of either of these syndromes alone. ${ }^{36}$ We hypothesised that SIB in TS is an expression of the intersection between two comorbid syndromes in TS: the presence of compulsivity plus impulse or affect dysregulation. We predicted that SIB in our TS study sample would be correlated with the presence of OC symptoms plus symptoms of impulse dysregulation, measured indirectly by presence of ADHD symptoms or risk taking behaviours, or with symptoms of affect dysregulation, as measured by the presence of an affective disorder or rage.

SIB is very heterogeneous, and can encompass behaviours as disparate as trichotillomania (compulsive hair pulling) and ritual self mutilation by burning or cutting. ${ }^{16}$ Although several schemas have been suggested for categorising SIB, there is currently no consistent classification system. The most widely used schema divides SIB into four major categories, including stereotypic, major, compulsive, and impulsive. ${ }^{17}$ Unfortunately, however, there is significant overlap between these categories in the types of SIB that are included, the severity of symptoms, and characteristics such as rate or pattern of behaviours. For this study, we used a simpler classification system, dividing SIB into moderate and severe categories. We hypothesised that this approach will be more clinically useful by reducing the overlap between categories and simplifying treatment decisions. In this schema, moderate SIB includes behaviours that result in moderate tissue damage, such as skin picking or scratching that leads to profuse bleeding, scarring, or infections requiring antibiotic treatment. Severe SIB includes extreme self injurious or mutilatory behaviours that lead to permanent, potentially impairing sequelae, such as self cutting, deliberate eye enucleation, or castration.

\section{METHODS \\ Subjects}

The study sample consisted of 297 individuals with TS aged 5-75 years, who were recruited for three genetic studies between 1996 and 2001, including a population genetic mapping (PGM) study in the Central Valley of Costa Rica, a PGM study among individuals of Ashkenazi Jewish descent in the US, and an affected sibling pair (ASP) study. Subjects in Costa Rica were recruited from a variety of sources, including referrals from healthcare professionals, advertisements in the national newspaper and television, assessments in schools, and response from family members who had heard of the study. Subjects in the US, for both the PGM and ASP studies, were recruited primarily from TS speciality clinics and via the Tourette Syndrome Association (TSA). Informed consent (and assent, for children older than 5 years) was obtained for all subjects, and the studies were all approved by the relevant institutional review boards.

\section{Diagnostic assessments}

For all three subject groups, diagnostic information was systematically gathered using structured instruments. The Yale Self Report Form (YSRF), a diagnostic instrument designed by the Genetic Consortium of the Tourette Syndrome Association, was administered to subjects and their parents by a psychiatrist with experience in the diagnosis of TS, and was used to elicit specific information about motor and vocal tics, OCD, SIB, and ADHD symptoms, and risk taking behaviours. ${ }^{18}$ The Yale Global Tic Severity Scale (YGTSS) was used to assess the severity of motor and phonic tics (on a scale of 1 to 15 ). ${ }^{19}$ Rage, which was defined as the presence of intermittent episodes of sudden, uncontrollable explosive or aggressive behaviours grossly out of proportion to provocation and atypical of the individual's usual personality, was assessed in all subjects using a 4 point questionnaire. ${ }^{20}$ Lifetime symptom prevalence and worst ever severity was assessed in all cases.

Information on developmental history, other comorbid disorders, and medical history was collected via a semistructured interview that contained specific questions regarding developmental and school milestones, medical conditions relevant to the genetic study (such as history of head trauma), medication use, and history of tic or related symptoms in each member of the immediate family and/or extended family. It also provided the interviewer with an opportunity to probe for other potentially relevant history, such as family history of unrelated psychiatric disorders or other medical problems. Because parents were generally present during the interview, we were able to confirm childhood symptoms reported by adults in the majority of cases. Each interview was videotaped for direct confirmation of tic symptoms. When available, medical records were obtained to elicit further medical and treatment history. Diagnoses of TS, OCD, ADHD, and other psychiatric diagnoses (see below) were made using all available materials according to DSM IV criteria by an experienced clinician (CAM).

Information sufficient to make DSM IV diagnoses of TS, OCD, and ADHD was obtained for all cases using the YSRF. Although information on other comorbid psychiatric disorders (depression, bipolar disorder) was collected during the clinical interviews in all cases, the presence of such comorbidities was systematically assessed using a structured diagnostic instrument in only a subset of study subjects $(n=76)$. The presence of psychiatric comorbidities other than OCD, ADHD, SIB, and rage was systematically assessed for all subjects in the ASP studies using the Kiddie Schedule for Affective Disorders or the Structured Clinical Interview for the Diagnostic and Statistical Manual, and for the first 30 subjects in the Costa Rican sample using the Spanish translation of the Diagnostic Interview Schedule for Children. ${ }^{21}$ Because of the design of the PGM genetic studies in Costa Rica and in the US, systematic information on comorbid psychiatric disorders using a structured interview was not available on the remainder of the PGM subjects. For this reason, all analyses involving comorbid diagnoses other than OCD, ADHD, and rage were performed only for the subset of subjects for whom complete diagnostic information was available.

\section{Statistical analysis}

\section{Outcome variables}

We examined two outcome variables: presence of SIB and presence of severe SIB. SIB was defined as deliberate, self directed behaviour that resulted in tissue damage or injury. Behaviours such as skin picking or scratching were included if they were severe enough to result in scarring, repeated infections requiring antibiotic treatment, or profuse bleeding, as was deliberate head banging. Motor tics such as head banging tics that resulted in inadvertent self injury were not included. Severe SIB was defined as a separate category, and included actions that could result in permanent impairing tissue damage such as self cutting with sharp instruments, deliberately poking in or at eyes in a way that could cause harm, sewing fingers together, and severe deliberate repetitive head banging that resulted in concussion.

\section{Statistics}

All statistical analyses were generated using Stata 6.0. The relationship between the outcome variables and each predictor variable of interest was initially assessed independently. $\chi^{2}$ analyses and Fisher's exact tests were used for 
categorical variables, and logistic regression was used for continuous variables. Each predictor variable that was significantly associated with SIB or severe SIB $(p \leqslant 0.05$, uncorrected) was then entered into a logistic regression. Potential confounding variables, such as sex, age, and study sample (Ashkenazi versus Costa Rican versus ASP) were also entered into the model. Variables of interest were added to the model sequentially, and were then individually removed until the model parameters were maximised. The best fit model was then compared with the larger initial model using a likelihood ratio test.

\section{RESULTS}

Because there were differences in how subjects from each of the studies were ascertained, leading to potential bias in the results, differences in severity between the groups were assessed. In general, the Costa Rican subjects were less severely affected than were the Ashkenazi or the ASP subjects, with a lower percentage of OCD and SIB, and lower motor and phonic tic severity scores than the other groups. The Costa Rican subjects also tended to be somewhat younger, and of a slightly higher socioeconomic status (table 1). These differences are most likely to be the result of an ascertainment bias: $47 \%$ of the Costa Rican subjects were recruited through media advertisements, family member referrals, or school referrals, whereas the vast majority of Ashkenazi and ASP subjects were recruited via healthcare professionals and the TSA (table 2). Because of the clear differences between the groups, logistic regressions were initially performed separately for each group. Results of the regressions were similar between groups (data not shown). Potential interactions between the presence of SIB or severe SIB and study group were assessed using a likelihood ratio test, which indicated that there were no significant interactions between study group and either SIB (likelihood ratio test statistic $=10.96, \mathrm{p}=0.69, \mathrm{df}=14$ ) or severe SIB (likelihood ratio test statistic $=7.65, \mathrm{p}=0.1 \mathrm{l}, \mathrm{df}=4$ ). Therefore, only the logistic regressions for the combined groups are presented, with the study group included as a term in the regression models.

\section{Prevalence of SIB}

Overall, $29 \%$ of TS subjects had SIB, and $4 \%$ had severe SIB (table 1). There were significant differences in rates of SIB between the subject groups, with the Ashkenazi subjects having the highest rate of SIB and the Costa Rican subjects the lowest. Rates of SIB differed significantly by age and referral source, although not by gender, with adults and those referred via a healthcare provider being more likely to have experienced SIB $\left(\chi^{2}=3.73, \mathrm{p}=0.05, \mathrm{df}=2\right.$ and
Table 2 Referral sources for TS study subjects in three genetic studies

\begin{tabular}{|c|c|c|c|c|}
\hline Referral source & $\begin{array}{l}\text { Ashkenazi } \\
\text { ( } n=132 \text { ) }\end{array}$ & $\begin{array}{l}\text { Costa Rican } \\
(n=119)\end{array}$ & $\begin{array}{l}\text { Sibling } \\
\text { pairs } \\
(n=46)\end{array}$ & $\begin{array}{l}\text { Total } \\
(n=297)\end{array}$ \\
\hline $\begin{array}{l}\text { Healthcare } \\
\text { professionals }\end{array}$ & $72.9(96)$ & $52.5(63)$ & & 53.5 (159) \\
\hline TSA & $23.3(31)$ & & $100(46)$ & $25.8(76)$ \\
\hline Family members & $3.8(5)$ & 3.33 (4) & & $3.0(9)$ \\
\hline Media & & $40(47)$ & & $16.0(47)$ \\
\hline Schools & & $4.2(5)$ & & $1.7(5)$ \\
\hline \multicolumn{5}{|c|}{$\begin{array}{l}\text { Values are } \%(n) \\
\chi^{2}=244.4, p<0.0001, d f=8\end{array}$} \\
\hline
\end{tabular}

$\chi^{2}=17.2, \mathrm{p}=0.002, \mathrm{df}=8$, respectively). The rate of severe SIB was higher among the sibling pair sample than among either of the other study groups, although because of the very small sample sizes this difference was not statistically significant. Rates of severe SIB were not significantly different by gender, age, or referral source, although as with SIB, there was a trend towards higher rates of severe SIB in subjects referred by medical professionals or the TSA.

Independent associations between SIB or severe SIB and each predictor variable are given in table 3. Because these associations were intended to determine the potentially relevant predictor variables for the logistic regression, they were not corrected for multiple testing. All of the OCD variables were significantly associated with SIB and severe SIB, as were several variables related to impulse control problems (such as rages). Although having a diagnosis of ADHD was associated with SIB, specific ADHD related symptom profiles, such as inattention or hyperactivity, were not. The starting logistic regression model for SIB included OC symptoms (presence of OCD, violent obsessions, violent/ aggressive compulsive behaviours, number of obsessive symptoms, and family history of obsessive symptoms), symptoms related to impulsivity (rages, risk taking behaviours, presence of $\mathrm{ADHD}$, and ADHD related impulsivity), presence of OCD plus ADHD, and motor and phonic tic severity and study sample, which were considered to be possible confounders $(\mathrm{n}=162$, likelihood ratio (LR) $\chi^{2}=43.29, \mathrm{p}=0.0001$, pseudo $\left.\mathrm{R}^{2}=0.21\right)$. This model is presented in table 4. Two variables, the presence of violent/ aggressive compulsive behaviours and the severity of motor tics, were particularly strongly associated with SIB. Other variables related to compulsivity (including the presence of OCD plus ADHD), although not independently statistically significant, were also positively correlated with SIB (odds ratio $>1$ ), whereas variables related to impulsivity alone

Table 1 Differences in sample characteristics between study groups

\begin{tabular}{|c|c|c|c|c|c|c|}
\hline & $\begin{array}{l}\text { Ashkenazi } \\
\text { ( } n=132 \text { ) }\end{array}$ & $\begin{array}{l}\text { Costa Rican } \\
(n=119)\end{array}$ & $\begin{array}{l}\text { Sibling pairs } \\
(n=46)\end{array}$ & $\begin{array}{l}\text { Total } \\
(n=297)\end{array}$ & $\begin{array}{l}\text { F statistic } \\
\text { ( } 2 \mathrm{df} \text { ) or } \chi^{2}\end{array}$ & $p$ value \\
\hline Male, $\%$ & 73.7 & 80.8 & 78.3 & 77.3 & 1.87 & 0.39 \\
\hline Mean (SD) age at interview, years & $22.5(15.0)$ & $15.8(11.9)$ & $20.4(14.7)$ & $19.5(14.1)$ & 7.47 & 0.0007 \\
\hline SES & $2.6(1.1)$ & $3.1(0.9)$ & 2.7 (1.3) & $2.8(1.1)$ & 3.47 & 0.03 \\
\hline \multicolumn{7}{|l|}{ Mean (SD) tic severity } \\
\hline Motor & $13.0(2.3)$ & $10.3(1.9)$ & $11.5(2.8)$ & $11.7(2.6)$ & 48.7 & $<0.0001$ \\
\hline Phonic & $11.7(3.2)$ & $8.1(2.4)$ & $10.1(2.4)$ & $10.0(3.4)$ & 47.0 & $<0.0001$ \\
\hline \multicolumn{7}{|l|}{ Psychiatric comorbidities, \% } \\
\hline OCD & 61.4 & 4.3 & 71.1 & 40.1 & 105.35 & $<0.0001$ \\
\hline $\mathrm{ADHD}$ & 43.9 & 23.5 & 69.6 & 39.7 & 31.1 & $<0.0001$ \\
\hline$O C D+A D H D$ & 34.6 & 3.3 & 58.7 & 25.8 & 63.1 & $<0.0001$ \\
\hline SIB & 43 & 14 & 28 & 29 & 25.3 & $<0.0001$ \\
\hline Severe SIB & 5 & 1 & 7 & 4 & 4.7 & $0.055^{\star}$ \\
\hline
\end{tabular}

Socioeconomic status (SES) is measured using the Hollingshead rating scale. ${ }^{27}$ Mean motor and phonic tic severity was measured using the YGTSS.

*Fisher $\mathrm{p}$ value. 
(including rages, ADHD, and ADHD related impulsivity) were not. Two smaller models were then tested by removing either the variables related to compulsivity or the variables related to impulsivity, and then were compared with the larger model. The OC model included presence of OCD, violent obsessions, violent/aggressive compulsive behaviours, number of obsessive symptoms, and OCD plus ADHD, as well as motor and vocal tic severity and study group ( $\mathrm{n}=162$, LR $\chi^{2}=40.37, \mathrm{p}<0.00001$, pseudo $\left.\mathrm{R}^{2}=0.20\right)$. This model was not significantly different than the overall model (LR $\left.\chi^{2}=2.92, p=0.40\right)$, suggesting that the OC variables were strongly correlated with SIB, and were the primary contributors to the significance of the overall model. The impulsive model included presence of ADHD, rages, risk taking behaviours, ADHD related impulsivity, motor and vocal tic severity, and study group $\left(\mathrm{n}=162, \mathrm{LR} \chi^{2}=31.11\right.$, $\mathrm{p}=0.0001$, pseudo $\left.\mathrm{R}^{2}=0.15\right)$. This model was marginally different from the overall model, although this difference did not quite reach statistical significance (LR $\chi^{2}=12.17$, $\mathrm{p}=0.058)$. When OCD plus ADHD was added back into the impulsive model $\left(\mathrm{n}=162\right.$, LR $\left.\chi^{2}=41.81, \mathrm{p}<0.00001\right)$, there was no difference between this model and the larger general model $\left(\right.$ LR $\left.\chi^{2}=1.48, p=0.83\right)$.

For severe SIB, the same procedure was followed. However, the overall model, which included the compulsivity and impulsivity variables, severity indices, and sample group, was not statistically significant $\left(\mathrm{n}=123, \mathrm{LR} \chi^{2}=20.05, \mathrm{df}=13\right.$, $\mathrm{p}=0.09$, pseudo $\mathrm{R}^{2}=0.34$ ), nor was the model that included only the compulsivity variables $\left(n=123, \mathrm{LR} \chi^{2}=9.68, \mathrm{df}=9\right.$, $\mathrm{p}=0.38$, pseudo $\left.\mathrm{R}^{2}=0.16\right)$. Although the impulsivity model was statistically significant overall $\left(\mathrm{n}=123\right.$, LR $\chi^{2}=18.38$, $\mathrm{df}=7, \mathrm{p}=0.01$, pseudo $\mathrm{R}^{2}=0.31$ ), the best fit model for severe SIB included only rages, risk taking behaviours, and severity of motor and phonic tics, all of which were positively correlated with the presence of severe SIB, and the Ashkenazi sample group, which was negatively associated with having severe SIB compared with the sibling pairs, which acted as the reference group $\left(\mathrm{n}=123, \mathrm{LR} \chi^{2}=18.31, \mathrm{df}=5, \mathrm{p}=0.003\right.$, pseudo $\mathrm{R}^{2}=0.31$ ) (table 5 ). The Costa Rican study group was not included in this regression because of small sample sizes (only one individual in this group had severe SIB).

The relationship between other psychiatric comorbidities such as mood disorders and SIB was also assessed. In the 76 subjects for whom complete data were available, affective disorders, conduct or other behavioural disorders, anxiety disorders other than OCD, and eating disorders were not significantly associated with either SIB or with severe SIB (table 5). For this reason, and because of the small sample sizes, these variables were not included in the logistic regressions.

\section{DISCUSSION}

Unexpectedly, there was little overlap in the predictors for SIB and those for severe SIB. For SIB, the most important predictor variables appeared to be related to OC symptoms. Contrary to our hypothesis, impulse/affect dysregulation, at least as indirectly measured here, was not significantly correlated with SIB. For severe SIB, the opposite was truethe most important predictor variables were related not to OC symptoms, but to tic severity and to the strongest clinical indicators of lack of impulse control (rages and risk taking behaviours).

These findings suggest that SIB and severe SIB may be different phenomena. In our study sample, SIB was primarily manifested as skin picking or scratching, hitting oneself, or hitting objects such as a wall. All of these behaviours, although somewhat more severe than expected, fall into the clinical symptom spectrum that lies between complex motor tics and compulsions. Seen in this context, it is not surprising that the best fit model for SIB primarily included variables

Table 3 Variables considered for entry into the logistic regression models and their corresponding independent significance levels for SIB and severe SIB

\begin{tabular}{|c|c|c|}
\hline Variable & SIB & Severe SIB \\
\hline Gender* & $\chi^{2}=0.778 ; p=0.780$ & Fisher; $p=1.00$ \\
\hline Age at interview (under or over 18 )* & $\chi^{2}=3.73 ; p=0.053$ & Fisher; $p=0.205$ \\
\hline Referral source & $\chi^{2}=17.2 ; p=0.002$ & Fisher; $p=0.571$ \\
\hline \multicolumn{3}{|l|}{ Tic severity } \\
\hline Motor & $Z=5.45 ; p<0.0001$ & $Z=2.14 ; p=0.032$ \\
\hline Phonic & $Z=4.93 p<0.0001$ & $Z=2.63 ; p=0.009$ \\
\hline \multicolumn{3}{|l|}{ Environmental and genetic factors } \\
\hline Prenatal problems* & $\chi^{2}=0.41 ; p=0.82$ & Fisher; $p=0.396$ \\
\hline Perinatal distress* & $\chi^{2}=1.43 ; p=0.49$ & Fisher; $p=0.75$ \\
\hline Family history of TS* & $\chi^{2}=0.28 ; p=0.60$ & Fisher; $p=0.695$ \\
\hline Bilineal family history of TS* & $\chi^{2}=0.64 ; p=0.42$ & Fisher; $p=0.282$ \\
\hline Family history of OCS & $\chi^{2}=14.07 ; p<0.0001$ & Fisher; $p=0.75$ \\
\hline \multicolumn{3}{|l|}{ Obsessive symptoms } \\
\hline OCD & Fisher; $p<0.0001$ & Fisher; $p=0.008$ \\
\hline Number of obsessive symptoms & $Z=5.28 ; p<0.0001$ & $Z=2.1 ; p=0.03$ \\
\hline Violent obsessions & $\chi^{2}=33.85 ; p<0.0001$ & Fisher; $p=0.028$ \\
\hline Violent/aggressive compulsive behaviours & $\chi^{2}=28.39 ; p<0.0001$ & Fisher; $p=0.005$ \\
\hline \multicolumn{3}{|l|}{ Attentional symptoms } \\
\hline ADHD & $\chi^{2}=5.19 ; p=0.023$ & Fisher; $p=0.121$ \\
\hline ADHD, impulsive symptoms & Fisher; $p=0.089$ & Fisher; $p=0.141$ \\
\hline ADHD, inattentive symptoms* & $\chi^{2}=3.71 ; p=0.16$ & Fisher; $p=0.163$ \\
\hline ADHD, hyperactive symptoms* & Fisher; $p=0.033$ & Fisher; $p=0.141$ \\
\hline \multicolumn{3}{|l|}{ Other psychiatric comorbidities } \\
\hline OCD+ADHD & $\chi^{2}=17.67 ; p<0.0001$ & $\chi^{2}=7.89 ; p=0.005$ \\
\hline Rages & $\chi^{2}=5.53 ; p=0.019$ & Fisher; $p=0.002$ \\
\hline Risk taking & Fisher; $p=0.02$ & Fisher; $p=0.122$ \\
\hline Anxiety disorders* & $\chi^{2}=0.48 ; p=0.49$ & Fisher; $p=1.000$ \\
\hline Mood disorders* & Fisher; $p=1.000$ & Fisher; $p=0.301$ \\
\hline Conduct disorders* & $\chi^{2}=1.23 ; p=0.27$ & Fisher; $p=0.133$ \\
\hline Eating disorders* & $\chi^{2}=0.027 ; p=0.87$ & Fisher; $p=0.214$ \\
\hline
\end{tabular}


Table 4 Logistic regression for SIB in TS, overall model

\begin{tabular}{|c|c|c|c|c|c|c|}
\hline \multirow[b]{2}{*}{ SIB } & \multirow[b]{2}{*}{ Odds ratio } & \multirow[b]{2}{*}{ SE } & \multirow[b]{2}{*}{$\mathbf{Z}$} & \multirow[b]{2}{*}{$\mathbf{P}>|\mathbf{z}|$} & \multicolumn{2}{|l|}{$95 \% \mathrm{Cl}$} \\
\hline & & & & & Lower & Upper \\
\hline OCD & 1.16 & 0.70 & 0.25 & 0.80 & 0.36 & 3.81 \\
\hline Violent obsessions & 1.47 & 0.74 & 0.77 & 0.44 & 0.55 & 3.96 \\
\hline $\begin{array}{l}\text { Violent/aggressive compulsive } \\
\text { behaviours }\end{array}$ & 4.27 & 2.30 & 2.70 & 0.007 & 1.49 & 12.25 \\
\hline Number of obsessive symptoms & 1.01 & 0.04 & 0.32 & 0.75 & 0.94 & 1.09 \\
\hline Family history of OCD & 1.08 & 0.49 & 0.17 & 0.86 & 0.44 & 2.65 \\
\hline ADHD & 0.44 & 0.38 & -0.96 & 0.34 & 0.08 & 2.36 \\
\hline Rages & 0.69 & 0.39 & -0.65 & 0.52 & 0.23 & 2.11 \\
\hline Risk taking behaviours & 2.05 & 1.13 & 1.30 & 0.19 & 0.69 & 6.06 \\
\hline ADHD related impulsivity & 0.98 & 0.50 & -0.04 & 0.97 & 0.36 & 2.65 \\
\hline OCD plus ADHD & 2.08 & 2.03 & 0.75 & 0.45 & 0.31 & 14.08 \\
\hline Severity of phonic tics & 1.01 & 0.08 & 0.14 & 0.89 & 0.86 & 1.18 \\
\hline Severity of motor tics & 1.29 & 0.17 & 2.05 & 0.04 & 1.01 & 1.67 \\
\hline Ashkenazi & 2.82 & 2.26 & 1.29 & 0.20 & 0.58 & 13.58 \\
\hline Costa Rican & 1.89 & 2.27 & 0.53 & 0.60 & 0.18 & 19.95 \\
\hline
\end{tabular}

related to compulsivity. Severe SIB, on the other hand, was correlated with impulsivity but not with compulsivity in our sample. This fits with the hypothesis that SIB in TS, as defined by more serious acts of self injury, may be related to dysregulation of affect, as has been postulated for SIB in other neuropsychiatric disorders. ${ }^{1216} 1723$

Other authors have also suggested that SIB in TS falls into distinct clinical categories. Robertson et al. argued that certain self injurious behaviours, such as repetitive hitting behaviours, represent compulsions, while the more complex forms of SIB such as eye poking are more likely to represent stereotypies. ${ }^{3}$ Although this suggestion is consistent with the classification system proposed by Favazza et al, ${ }^{13}{ }^{17}$ in practice, separating compulsive from stereotypic behaviour can be difficult in the TS population, in part because of the difficulty in distinguishing between complex tics or stereotypies and certain types of compulsions such as compulsive touching or picking. Treatment practices for those symptoms that fall into the grey area between complex tics and compulsions reflect this; treatment typically includes both a serotonin reuptake inhibitor and an atypical anti-psychotic. We hypothesised that from a clinical practice perspective, classification and treatment based on severity of the self injurious behaviour would be a simpler and more accessible approach than trying to distinguish accurately between such complexities. This was borne out by the data: in our classification system, moderate SIB encompassed symptoms from both the compulsive and stereotypic categories proposed by Favazza et al. and accounted for the majority of SIB in TS, while severe SIB encompassed the major and impulsive categories, and was more consistent with the prevalence of SIB in the general psychiatric population.

The majority of studies examining SIB in TS have not differentiated severe from mild to moderate types of self injurious behaviours, which may account for the high prevalence estimates and the variability across studies. In our study, severe SIB was present in only $4 \%$ of subjects, which is consistent with the rates seen in the general psychiatric population. ${ }^{1}$ Milder SIB was present in a much higher proportion of subjects (up to $43 \%$ ). The rates of SIB in each of our study samples correlated with the overall TS severity levels in each group, and differences between the groups are likely to be due to differences in ascertainment rather than other factors such as ethnicity. In general, subjects in the Costa Rican TS sample were the least severely affected, both in terms of OC symptoms and in terms of tic severity, and also had the lowest rates of SIB, whereas the Ashkenazi subjects were more severely affected, and had the highest rates of SIB. The Costa Rican sample was primarily recruited from the general population, and is thus composed of individuals with a wide range of symptoms, including very mild symptoms, whereas the Ashkenazi sample was recruited almost exclusively from physicians' offices, and the vast majority were referred by psychiatrists at tertiary care clinics specialising in the diagnosis and treatment of TS.

Although no conclusive statements regarding the nature of SIB in TS can be drawn from this work, it does lead to some interesting suppositions. If it is true that mild SIB in TS is primarily compulsive, for example, the optimal treatment for behaviours such as persistent skin picking may be the treatments traditionally reserved for OCD, including the use of serotonin reuptake inhibitors, perhaps in conjunction with atypical antipsychotics, and cognitive behavioural therapy directed specifically at reducing the behaviour. Serotonergic agents have been shown to be effective in reducing SIB in mental retardation and in autism, as have the atypical neuroleptics (reviewed by Villalba \& Harrington ${ }^{24}$ ). These medications have also been found to be helpful in disorders thought to be related to TS, OCD, and SIB, such as trichotillomania and skin picking. ${ }^{4}$

Table 5 Logistic regression for severe SIB in TS, best fit model $\left(n=123, \operatorname{LR} \chi^{2}=18.31\right.$, $d f=5, p=0.003$, pseudo $R^{2}=0.31$ )

\begin{tabular}{|c|c|c|c|c|c|c|}
\hline \multirow[b]{2}{*}{ Severe SIB } & \multirow[b]{2}{*}{ Odds Ratio } & \multirow[b]{2}{*}{ SE } & \multirow[b]{2}{*}{ z } & \multirow[b]{2}{*}{$P>|z|$} & \multicolumn{2}{|l|}{$95 \% \mathrm{Cl}$} \\
\hline & & & & & Lower & Upper \\
\hline Rages & 12.87 & 12.79 & 2.57 & 0.01 & 1.84 & 90.30 \\
\hline Risk-taking & 1.64 & 1.49 & 0.55 & 0.59 & 0.28 & 9.68 \\
\hline Severity of motor tics & 1.22 & 0.41 & 0.59 & 0.56 & 0.63 & 2.37 \\
\hline Severity of phonic tics & 1.52 & 0.47 & 1.35 & 0.18 & 0.83 & 2.79 \\
\hline Ashkenazi & 0.06 & 0.08 & -2.15 & 0.03 & 0.005 & 0.78 \\
\hline
\end{tabular}


Severe SIB presents a more difficult problem, and can be approached in two ways. Non-repetitive forms of severe SIB such as those commonly seen in patients with mood disorders or personality disorders, and exemplified by the patient in our sample who sewed his fingers together, appear to be related to problems with impulse or affective dysregulation, and may be most effectively treated as such with mood stabilisers. It has been shown that in adults with severe, long lasting SIB and aggression, and in patients with Lesch-Nyhan syndrome, agents such as divalproex and gabapentin have been effective in reducing SIB. ${ }^{24}$ For severe repetitive SIB such as repeated eye poking or very severe skin picking, on the other hand, the opiate blocker naltrexone may be the treatment of choice. Severe repetitive SIB is hypothesised by some to be caused by abnormalities in sensation at the body site, perhaps due to dysregulation in the opiate (endorphin/encephalin) system. A relationship between altered pain sensitivity and SIB or self mutilation has been observed in patients with schizophrenia, borderline personality disorder, and Alzheimer's disease. ${ }^{24}$ Reduced sensation to pain and decreases in sensory nerve transmission have both been documented in subjects with SIB, particularly in those with repetitive skin picking. ${ }^{4}$ Between $30 \%$ and $70 \%$ of patients with SIB have been shown to have dysregulation in the opioid system or in the pro-opiomelanocortin system, which includes the neuropeptides $\beta$ encephalin, adrenocortictropic hormone, and melanocyte stimulating hormone. Repetitive SIB is associated with an elevation in the mean levels of plasma metenkephalin and an increase in $\beta$-endorphin secretion immediately after an SIB episode. In some patients with chronic SIB, the $\beta$-endorphin response is uncoupled from the ACTH response, which is thought to protect against opiate tolerance. ${ }^{24}$ It has been hypothesised that unopposed $\beta$-endorphin secretion leads to tolerance and the subsequent reinforcement of SIB. ${ }^{24}$ In a study of patients with mental retardation, elevated plasma levels of $\beta$-endorphin following an SIB episode predict subsequent response to naltrexone, with those having the greatest post-episode $\beta$-endorphin levels showing the greatest improvement. ${ }^{44}$

In non-verbal patients with mental retardation and severe SIB who responded to naltrexone, the body sites targeted the most frequently for SIB were associated with alterations in skin temperature compared with other body sites. ${ }^{425}$ Sensory symptoms, often described by adults as "sensory tics" or "premonitory urges" and by children as an "itch-like feeling", are well known phenomena in TS. ${ }^{26}$ It is possible that in some cases these sensory phenomena are related to the occurrence of SIB, perhaps through persistent alterations in either skin temperature or pain perception or both. Although we assessed subjects for the general presence or absence of premonitory sensory phenomena, unfortunately we do not have information regarding the relationship between such phenomena and SIB in our study samples. To date, there are no data on the physical correlates of SIB in TS, although this work has been performed in patients with mental retardation and autism or other pervasive developmental disorders. It would be interesting to learn if TS patients with SIB had abnormalities in skin temperature or in sensory nerve transmission, compared with TS patients without SIB and with those with autism or other disorders in which SIB is common.

\section{Limitations}

The primary limitations of this study relate to the method of data collection employed. Clinical data were collected retrospectively from subjects and their family members in three different population groups, leading to potential bias, including recall and ascertainment bias. As far as possible, any ascertainment bias was controlled for in the analyses. Where relevant, data for each group are reported separately. As noted, where there were no significant differences between the groups, pooled data are presented.

In addition, longitudinal data were not available: as noted in the Methods section, only information about lifetime symptom prevalence and worst ever symptom severity was available. For this reason, it is not possible to assess whether the SIB was temporally related to the presence of obsessive or impulsive symptoms. Similarly, data are not available regarding the age of occurrence of SIB. Tic severity is known to decrease with age in the majority of individuals with TS. ${ }^{28}$ Although SIB appears to persist throughout life in mentally retarded and autistic individuals, the longitudinal time course of SIB in TS is unknown, and would be important to investigate. $^{29}$

\section{ACKNOWLEDGEMENTS}

This study was supported by grants from the NINDS (R0I 444653 and R01 NS40024), the NIMH (K02 MH01375), and the NCRR (K23 RR15533). We thank the patients and families participating in the study, the Tourette Syndrome Association, the Tourette Syndrome Association International Consortium for Genetics, and G Klein for their assistance in collecting the data.

\section{Authors' affiliations}

C A Mathews, J Waller, Department of Psychiatry, University of California, San Diego, La Jolla, CA, USA

D Glidden, Department of Epidemiology and Biostatistics, University of California, San Francisco, San Francisco, CA, USA

T L Lowe, Department of Psychiatry, Division of Child and Adolescent Psychiatry, University of California, San Francisco, San Francisco, CA, USA

L D Herrera, Departments of Psychiatry and Pediatrics, University of Costa Rica School of Medicine, San José, Costa Rica

C L Budman, R D Bruun, Department of Psychiatry, North Shore University Hospital and New York University School of Medicine, Manhasset, New York, USA

G Erenberg, Department of Neurology, Cleveland Clinic Foundation, Cleveland, $\mathrm{OH}$, USA

A Naarden, Department of Neurology, University of Texas Southwestern Medical School, Dallas, TX, USA

N B Freimer, Departments of Psychiatry and Human Genetics, University of California, Los Angeles, Los Angeles, CA, USA

V I Reus, Department of Psychiatry, University of California, San Francisco, CA, USA

Competing interests: none declared

\section{REFERENCES}

1 Herpertz S. Self-injurious behavior: psychopathological and nosological characteristics in subtypes of self-injurers. Acta Psychiatr Scand 1995;91:57-68.

2 Haw C, Hawton K, Houston K, et al. Psychiatric and personality disorders in deliberate self-harm patients. Br J Psych 2001;178:48-54

3 Robertson MM, Trimble MR, Lees AJ. Self-injurious behavior and the Gilles de la Tourette syndrome: a clinical study and review of the literature. Psychol Med 1989;19:611-25.

4 Schroeder SR, Oster-Granite ML, Berkson G, et al. Self-injurious behavior: gene-brain-behavior relationships. Ment Retard Develop Disabil Res Rev 2001;7:3-12.

5 American Psychiatric Association. Diagnostic and statistical manual of mental disorders, (DSM-IV), 4th ed. Washington DC: American Psychiatric Association Press, 1994.

6 Freeman RD, Fast DK, Burd L, et al. An international perspective on Tourette syndrome: selected findings from 3500 individuals in 22 countries. Dev Med Child Neurol 2000;42:436-47.

7 Eisenhauer GLL, Woody RC. Self-mutilation and Tourette's disorder. J Child Neurol 1987;2:265-7

8 Berthier ML, Campos VM, Kulisevsky J. Echopraxia and self-injurious behavior in Tourette's syndrome. Neuropsychiatry Neuropsychol Behav Neurol 1996;9:280-3

9 Micheli F, Gatto M, Gershanik O, et al. Gilles de la Tourette syndrome: clinical features of 75 cases from Argentina. Behav Neurol 1995;8:75-80.

10 Cath DC, Spinhoven P, Hoogduin CAL, et al. Repetitive behaviors in Tourette's syndrome and OCD with and without tics: what are the differences? Psychiatry Res 2001;101:171-85. 
11 Robertson MM, Gourdie A. Familial Tourette's syndrome in a large British pedigree: associated psychopathology, severity and potential for linkage analysis. Br J Psych 1990;156:515-21.

12 Nixon MK, Cloutier PF, Aggarwal S. Affect regulation and addictive aspects of repetitive self-injury in hospitalized adolescents. J Am Acad Child Adolesc Psychiatry 2002;41:1333-41.

13 Favazza AR, Rosenthal RJ. Diagnostic issues in self-mutilation. Hosp Community Psychiatry 1993;44:134-40

14 Walkup JT, Khan S, Schuerholz L, et al. Phenomenology and natural history of tic-related ADHD and learning disabilities. In: Leckman JF, Cohen DJ, eds. Tourette's Syndrome: tics, obsessions, compulsions. New York: John Wiley and Sons, 1999:63-79.

15 King RA, Leckman JF, Scahill L, et al. Obsessive-compulsive disorder, anxiety, and depression. In: Leckman JF, Cohen DJ, eds. Tourette's syndrome: tics, obsessions, compulsions. New York: John Wiley and Sons, 1999:43-62.

16 Simeon D, Favazza A. Self-injurious behaviors: phenomenology and assessment. In: Simeon D, Hollander E, eds. Self-injurious behaviors: assessment and treatment. Washington DC: American Psychiatric Press, 2000:1-28.

17 Favazza AR, Simeon D. Self-mutilation. In: Hollander E, Stein DJ, eds. Impulsivity and aggression. Chichester, England: John Wiley and Sons, 1995: 185-200.

18 The Tourette Syndrome Association International Consortium for Genetics. A complete genome screen in sib pairs affected by Gilles de la Tourette syndrome. Am J Hum Genet 1999:65:1428-36.

19 Leckman JF, Riddle MA, Hardin MT, et al. The Yale Global Tic Severity Scale: initial testing of a clinician-rated scale of tic severity. J Am Acad Child Adolesc Psychiatry 1989;28:566-73.
20 Budman CL, Rockmore L, Stokes J, et al Clinical phenomenology of episodic rage in children with Tourette Syndrome. J Psychosom Res 2003;55:59-65.

21 Bravo M, Woodbury-Fariña M, Canino GJ, et al. The Spanish translation and cultural adaptation of the Diagnostic Interview Schedule for Children (DISC) in Puerto Rico. Culture, Med Psychiatry 1993;17:329-44.

22 Bodfish JW, Symons FJ, Parker DE, et al. Varieties of repetitive behavior in autism: comparisons to mental retardation. J Autism Dev Disord 2000; 30:237-43

23 Haw C, Houston K, Townsend E, et al. Deliberate self harm patients with depressive disorders: treatment and outcome. J Affect Disord 2002;70:57-65.

24 Villalba R, Harrington CJ. Repetitive self-injurious behavior: a neuropsychiatric perspective and review of pharmacologic treatments. Semin Clin Neuropsychiatry 2000;5:215-26.

25 Symons FJ, Sutton KA, Bodfish JW. Preliminary study of altered skin temperature at body sites associated with self-injurious behavior in adults who have developmental disabilities. Am J Ment Retard 2001;106:336-43.

26 Miguel EC, de Rosario-Campos MC, Prado HS, et al. Sensory phenomena in obsessive-compulsive disorder and Tourette's disorder. J Clin Psychiatry 2000;61:150-6.

27 Hollingshead AB. Four factor index of social status. New Haven, CT: Yale University Department of Sociology, 1975

28 Leckman JF, Zhang H, Vitale A, et al. Trajectories of tic severity in Tourette's syndrome: the first two decades. Pediatrics 1998;102:14-19.

29 Schroeder SR, Mulick JA, Rojahn J. The definition, taxonomy, epidemiology, and ecology of self-injurious behavior. J Autism Devel Dis 1980;10:417-32. 